\title{
The Structure of Groups of Motions Admitted by Einstein-Maxwell Space-Times
}

\author{
M. L. Woolley \\ Institute of Physics, University of Bologna, Bologna, Italy
}

Received October 16, 1972; in revised form January 15, 1973

\begin{abstract}
The known symmetry of the non-null electromagnetic field, which acts as the source of a four-dimensional space-time satisfying the Einstein-Maxwell equations, is used to show that when such a space-time admits a group of motions, generated by a Killing vector, the structure constants for the group must satisfy an additional relation to the known relations of group theory.
\end{abstract}

\section{Introduction}

The work of Rainich (1925), and subsequently Misner and Wheeler (1957), has shown that in the absence of sources the equations of electromagnetism and gravitation can be expressed in a purely geometric form. A consequence of this was shown by Misner and Wheeler to be that the non-null electromagnetic field tensor $F_{\mu \nu}$ is determined up to a duality rotation by the metric tensor $g_{\mu v}$.

In the work which follows we shall see that this leads to the conclusion that when a four dimensional vacuum space-time, having a non-null electromagnetic field as its source, admits a group of motions generated by a Killing vector the infinitesimal transformations $\underset{v}{\mathscr{L}} F_{\mu \nu}$ of the electromagnetic field tensor $F_{\mu \nu}$ must be such that one of the equations

$$
\underset{v}{\mathscr{L}} F_{\mu \nu}=0
$$

or

$$
\mathscr{L}_{v}^{2} F_{\mu \nu}=-F_{\mu \nu}
$$

is satisfied. This has the consequence that the structure constants $c_{\alpha \beta}^{\gamma}$ for the group of motions must satisfy an additional relation to the known relations of group theory. 


\section{The Infinitesimal Transformations}

We will consider a four dimensional space-time which satisfies the Einstein-Maxwell equations. These may be written

and

$$
\left.\begin{array}{c}
R_{\mu \nu}-\frac{1}{2} g_{\mu \nu} R=4 \pi\left\{F_{\mu \sigma} F_{v}^{\sigma}+{ }^{*} F_{\mu \sigma}{ }^{*} F_{v}^{\sigma}\right\} \\
\frac{1}{\sqrt{-g}} \frac{\partial}{\partial x^{\nu}}\left\{\sqrt{-g} F^{\mu \nu}\right\}=0 \\
\frac{1}{\sqrt{-g}} \frac{\partial}{\partial x^{\nu}}\left\{\sqrt{-g}{ }^{*} F^{\mu \nu}\right\}=0
\end{array}\right\}
$$

where $F_{\mu \nu}$ is the electromagnetic field tensor and ${ }^{*} F^{\mu v}$ is its dual. We will assume that $F_{\mu \nu}$ is not null so that

and

$$
\left.\begin{array}{c}
F_{\mu \nu} F^{\mu v} \neq 0 \\
F_{\mu \nu} * F^{\mu v} \neq 0 .
\end{array}\right\}
$$

The mixed energy momentum tensor for the electromagnetic field has vanishing trace so that (2.1) may be written

$$
R_{\mu \nu}=4 \pi\left\{F_{\mu \sigma} F_{v}^{\sigma}+{ }^{*} F_{\mu \sigma}{ }^{*} F_{v}^{\sigma}\right\} .
$$

We will now consider that our space-time admits an $r$-parameter group of motions which is generated by a Killing vector. This requires that there exist $r$ linearly independent vectors $v_{\alpha}^{\sigma}$ which satisfy the equations of Killing. These may be written (Yano, 1955)

$$
\underset{v}{\mathscr{L}} g_{\mu \nu}=v^{\sigma} \frac{\partial}{\partial x^{\sigma}} g_{\mu \nu}+g_{\mu \sigma} \frac{\partial v^{\sigma}}{\partial x^{\nu}}+g_{\sigma \nu} \frac{\partial v^{\sigma}}{\partial x^{\mu}}=0
$$

and for each Killing vector $v_{\alpha}^{\sigma}$ we have an infinitesimal operator

$$
\underset{\substack{v \\ \alpha}}{\mathscr{L}} \equiv \underset{\alpha}{\mathscr{L}}
$$

such that $(2.5)$ is satisfied.

If we denote any of the independent vectors $v_{\alpha}^{\sigma}$ by $v^{\sigma}$ we find that the infinitesimal transformations $\underset{v}{\mathscr{L}} F_{\mu \nu}$ of the electromagnetic field tensor $F_{\mu \nu}$ must have the forms

and

$$
\left.\begin{array}{l}
\underset{v}{\mathscr{L}} F_{\mu \nu}=\frac{\partial A_{\mu}}{\partial x^{v}}-\frac{\partial A_{v}}{\partial x^{\mu}} \\
\underset{v}{\mathscr{L}} F^{\mu \nu}=\frac{\varepsilon_{\mu \nu \sigma \tau}}{\sqrt{-g}} \frac{\partial B_{\tau}}{\partial x^{\sigma}}
\end{array}\right\}
$$


where the two vectors $A_{\sigma}$ and $B_{\sigma}$ are defined by

and

$$
\left.\begin{array}{l}
A_{\sigma}=v^{v} F_{\sigma v} \\
B_{\sigma}=v^{v^{*}} F_{\sigma v}
\end{array}\right\}
$$

respectively. From the relations (2.7) we find that $\underset{v}{\mathscr{L}} F_{\mu \nu}$ satisfies Maxwell's Eqs. (2.2). We may use this fact in order to determine firstly the infinitesimal mode of transformation of the non-null field and subsequently the structure of the group of motions.

There are two distinct cases which have to be considered. Firstly it is possible for $\underset{v}{\mathscr{L}} F_{\mu \nu}$ to vanish. This is certainly a solution to the vacuum Maxwell equations and moreover the first integrability condition

$$
\underset{v}{\mathscr{L}} R_{\mu \nu \sigma \tau}=0
$$

of Killing's equation ensures that $\underset{v}{\mathscr{L}} R_{\mu \nu}=0$. It is therefore possible for a field satisfying

$$
\underset{v}{\mathscr{L}} F_{\mu \nu}=0
$$

to be a solution of the Einstein-Maxwell equations. The condition (2.10) expresses the invariance of the electromagnetic field under the action of the transformations generated by the Killing vector $v^{\sigma}$. We shall now consider the second case in which (2.10) is not satisfied.

When the electromagnetic field is not invariant its Lie derivative, with respect to the Killing vector $v^{\sigma}$, is a non-trivial solution of Maxwell's equations (2.2) with the metric tensor $g_{\mu v}$. Thus if

$$
f_{\mu \nu}=\underset{v}{\mathscr{L}} F_{\mu \nu}
$$

this electromagnetic field must have an energy-momentum tensor

$$
T_{\mu \nu}=-\frac{1}{2}\left(f_{\mu \sigma} f_{v}^{\sigma}+*_{\mu \sigma} f_{v}^{\sigma}\right)
$$

and Einstein's theory of gravitation requires that

$$
G_{\mu \nu}=-8 \pi T_{\mu \nu} .
$$

But in a four-dimensional space-time the Einstein tensor $G_{\mu \nu}$ is unique to within the cosmological term (e.g. Lovelock, 1971 and 1972) - which we are not considering here. Thus (2.13) requires that

$$
R_{\mu \nu}=4 \pi\left(f_{\mu \sigma} f_{v}^{\sigma}+{ }^{*} f_{\mu \sigma}{ }^{*} f_{v}^{\sigma}\right)
$$


and we may use this relation together with (2.4) in order to determine the precise relation between $f_{\mu \nu}$ and $F_{\mu \nu}$ in this case.

If we define the complex fields $\Gamma_{\mu \nu}$ and $\gamma_{\mu \nu}$ by

and

$$
\left.\begin{array}{l}
\Gamma_{\mu \nu}=F_{\mu \nu}+i^{*} F_{\mu \nu} \\
\gamma_{\mu \nu}=\mathscr{L}_{v} \Gamma_{\mu \nu}
\end{array}\right\}
$$

respectively then (2.4) and (2.14) may be written

and

$$
\left.\begin{array}{l}
R_{\mu \nu}=4 \pi \Gamma_{\mu \sigma} \bar{\Gamma}_{v}^{\sigma} \\
R_{\mu \nu}=4 \pi \gamma_{\mu \sigma} \bar{\gamma}_{v}^{\sigma}
\end{array}\right\}
$$

respectively where the bar denotes the complex conjugate. In addition, the theorem of Misner and Wheeler (1957) asserts that there exists an $\varepsilon$ such that

or

$$
f_{\mu \nu}=F_{\mu \nu} \operatorname{Cos} \varepsilon+{ }^{*} F_{\mu \nu} \operatorname{Sin} \varepsilon
$$

$$
\gamma_{\mu \nu}=e^{-i \varepsilon} \Gamma_{\mu \nu} .
$$

But

implies that

$$
\underset{v}{\mathscr{L}} R_{\mu \nu}=0
$$

and this yields

$$
\begin{gathered}
\Gamma_{\mu \sigma} \bar{\gamma}_{\nu}^{\sigma}+\gamma_{\mu \sigma} \bar{\Gamma}_{\nu}^{\sigma}=0 \\
R_{\mu \nu} \operatorname{Cos} \varepsilon=0
\end{gathered}
$$

which, since $R_{\mu \nu} \neq 0$, requires that $\varepsilon$ has the values $\left(\frac{\pi}{2}+2 m \pi\right)$ or $\left(\frac{3 \pi}{2}+2 m \pi\right)$ only where $m$ is any integer ${ }^{1}$. On substituting this in (2.18) and equating real and imaginary parts we conclude that

and

$$
\left.\begin{array}{c}
\underset{v}{\mathscr{L}} F_{\mu \nu}= \pm * F_{\mu \nu} \\
\underset{v}{\mathscr{L}} * F_{\mu \nu}=\mp F_{\mu \nu}
\end{array}\right\}
$$

represent the infinitesimal mode of transformation of the non-null noninvariant electromagnetic field.

The two relations (2.22) are equivalent to

$$
\mathscr{L}_{v}^{2} F_{\mu v}+F_{\mu v}=0
$$

which, when the Killing vector is known, is a second order linear partial differential equation for the functional form of $F_{\mu \nu}$ which satisfies the Einstein-Maxwell equations. As an example, if the Killing vector has

${ }^{1}$ I would like to thank Professor A. H. Taub for pointing out this short-cut in the calculation. M.L.W. 
the form $\delta_{(k)}^{\sigma},(2.23)$ reduces to

$$
\frac{\partial^{2} F_{\mu \nu}}{\partial x^{k 2}}+F_{\mu \nu}=0
$$

and its solution, which must satisfy (2.22), has the form

where

$$
\left.\begin{array}{rl}
F_{\mu \nu} & =a_{\mu \nu} \operatorname{Cos} \theta+* a_{\mu \nu} \operatorname{Sin} \theta \\
\theta & =x^{k}+\varphi
\end{array}\right\}
$$

and both $a_{\mu \nu}$ and $\phi$ are independent of $x^{k}$.

A physical interpretation of (2.23) is obtained if we consider the vectors $A_{v}$ and $B_{v}$ which give the local electric and magnetic fields respectively of a test observer who follows a path everywhere tangent to the Killing vector $v^{\sigma}$. Then (2.23) shows that the observer will find these field vectors rotating as he moves along and, for example, when $x^{k}$ has the role of a time co-ordinate this would amount to a rotation in time of the local field vectors.

We have now seen that if a source-free four dimensional space-time has integrable equations of Killing the non-null electromagnetic field must transform as either (2.10) or (2.22) and we are in a position to see what implications those relations have for the structure of the corresponding of motions.

\section{The Structure Relations}

In arriving at the relations (2.10) and (2.22) we considered an arbitrary member $v^{\sigma}$ of the set of $r$ generators for the group of motions. It follows that either (2.10) or (2.22) must be satisfied by each of the infinitesimal operators $\underset{\alpha}{\mathscr{L}}$ for the group in question. We will now consider this fact in more detail.

The infinitesimal operators $\underset{\alpha}{\mathscr{L}}$ can be shown to obey the commutation relations (Yano, 1955, p. 29)

where

$$
[\underset{\alpha}{\mathscr{L}}, \underset{\beta}{\mathscr{L}}] G_{\Lambda}=c_{\alpha \beta}^{\gamma} \underset{\gamma}{\mathscr{L}} G_{\Lambda}
$$

$$
\left[\begin{array}{c}
\mathscr{L} \\
\alpha
\end{array} \underset{\beta}{\mathscr{L}}\right] \equiv \underset{\alpha}{\mathscr{L}} \underset{\beta}{\mathscr{L}}-\underset{\beta}{\mathscr{L}} \underset{\alpha}{\mathscr{L}}
$$

and the $c_{\alpha \beta}^{\gamma}$ are the fundamental structure constants of the group. The quantity $G_{A}$ in (3.1) represents any linear differential geometric object.

We will now replace $G_{A}$ in (3.1) with $F_{\mu \nu}$ and consider these relations together with (2.10) and (2.22) - one of which must be satisfied by each $\underset{\alpha}{\mathscr{L}}$ when it acts on $F_{\mu v}$. 
Firstly we define the set $S_{I}$ of independent vectors $v_{\alpha}^{\sigma}$ which generate the invariance group of $F_{\mu v}$. Thus

$$
S_{I}=\left\{v_{\alpha}^{\sigma} \mid \mathscr{L}_{\alpha} F_{\mu \nu}=0\right\}
$$

and $S_{I}$ is clearly a subset of the set of generators for the group of motions.

A consideration of the right hand side of (3.1) shows that this becomes

where

$$
c_{\alpha \beta}^{\gamma} \underset{\gamma}{\mathscr{L}} F_{\mu \nu}= \pm c_{\alpha \beta}{ }^{*} F_{\mu \nu}
$$

$$
c_{\alpha \beta}=\sum_{\substack{\gamma \\ v \notin S_{I}}} c_{\alpha \beta}^{\gamma} .
$$

In considering the left hand side of (3.1) there are three distinct cases which we must take into account separately. These correspond to whether or not both, one or none of $\underset{\alpha}{ }$ and $v_{\beta}$ are contained in $S_{I}$.

If both of $v_{\alpha}$ and $v_{\beta}$ are in $S_{I}$ then the left hand side of (3.1) must vanish since $\underset{\alpha}{\mathscr{L}} F_{\mu \nu}$ and $\mathscr{L}_{\beta}^{\beta} F_{\mu \nu}$ both vanish. On the other hand, if one of $\underset{\alpha}{v}$ and $\underset{\beta}{v}$ is in $S_{I}$ the other must be such that

$$
\underset{v}{\mathscr{L}} F_{\mu \nu}= \pm * F_{\mu v}
$$

and, since the invariance of $F_{\mu \nu}$, under the transformations generated by a Killing vector, implies the invariance of its dual we see that the left hand side of (3.1) vanishes in this case too. Finally, if neither of $v$ and $v$ are contained in $S_{I}$ they must both be such that (3.6) is true. We $\alpha \quad \beta$ must then have

$$
\begin{aligned}
\underset{\alpha}{\mathscr{L}} \underset{\beta}{\mathscr{L}} F_{\mu \nu} & = \pm \underset{\alpha}{\mathscr{L}} * F_{\mu \nu} \\
& =-F_{\mu \nu}
\end{aligned}
$$

and, since this result is independent of the order of operation of $\underset{\alpha}{\mathscr{L}}$ and $\underset{\beta}{\mathscr{L}}$ it follows that the left hand side of (3.1) must again vanish.

We have now seen that if $F_{\mu \nu}$ satisfies (2.10) and (2.22) the left hand side of (3.1) must vanish. This means that the right hand side must vanish also, since (3.1) is an identity for a given group of motions. The only conclusion which may now be reached is that the quantity $c_{\alpha \beta}$ defined by (3.5) must vanish, i.e. we must have

$$
\sum_{\substack{\gamma \\ v \notin S_{I} \\ \gamma}} c_{\alpha \beta}^{\gamma}=0 .
$$

This relation is purely a consequence of the possible symmetry of the non-null Einstein-Maxwell electromagnetic field as expressed by the relations (2.10) and (2.22). 


\section{Conclusions}

It is well known that the structure constants $c_{\alpha \beta}^{\gamma}$ for an $r$-parameter group of motions must satisfy the relations

and

$$
c_{\alpha \beta}^{\gamma}+c_{\beta \alpha}^{\gamma}=0
$$

$$
c_{\alpha \beta}^{\gamma} c_{\gamma \delta}^{\sigma}+c_{\delta \alpha}^{\gamma} c_{\gamma \beta}^{\sigma}+c_{\beta \delta}^{\gamma} c_{\gamma \alpha}^{\sigma}=0 \text {. }
$$

We have seen in the work here that for a group of motions which leave unchanged the metric tensor $g_{\mu \nu}$ of a four dimensional vacuum EinsteinMaxwell spacetime, having a non-null electromagnetic field $F_{\mu \nu}$ as its source, we must consider in addition the relations (3.9). These, together with the further relations

$$
\begin{aligned}
\mathscr{L}_{v} F_{\mu \nu} & =0 \\
\mathscr{L}_{v}^{2} F_{\mu \nu} & =-F_{\mu \nu}
\end{aligned}
$$

and

$$
\stackrel{\mathscr{L}}{v} g_{\mu \nu}=0
$$

should allow a complete group theoretical characterization, of this class of electromagnetic space-times, to be carried out.

Acknowledgements. The author would like to thank an unknown referee for the Institute of Physics, in England, who's original suggestion led to the work presented here.

This work was carried out during a Royal Society European Programme Fellowship at the University of Bologna and financed by the Accademia Nazionale dei Lincei.

\section{References}

Lovelock, D.: Journal of Math. Phys. 12, 498 (1971).

Lovelock, D.: Journal of Math. Phys. 13, 874 (1972).

Misner, C. W., Wheeler, J. A. : Annals of Phys. 2, 525 (1957).

Rainich, G. Y.: Trans. Amer. Math. Soc. 27, 106 (1925).

Yano, K.: Theory of Lie derivatives and its application. Amsterdam: North-Holland 1955.

M. L. Woolley

Institute of Physics

University of Bologna

Via Irnerio 46, Bologna, Italy
M. L. Woolley's present address:

96 Highdown Road

Hove BN3 6EA Sussex, U.K. 
